\title{
Threshold dynamics of a stochastic SIVS model with saturated incidence and Lévy jumps
}

\author{
Yuanlin $\mathrm{Ma}^{1}$ and Xingwang $\mathrm{Yu}^{2^{*}}$ (D)
}

"Correspondence:

xwyu2006@126.com

${ }^{2}$ School of Management

Engineering, Zhengzhou University

of Aeronautics, Zhengzhou, China

Full list of author information is

available at the end of the article

\section{Springer}

\begin{abstract}
In this paper, we propose and analyze a stochastic SIVS model with saturated incidence and Lévy jumps. We first prove the existence of a global positive solution of the model. Then, with the help of semimartingale convergence theorem, we obtain a stochastic threshold of the model that completely determines the extinction and persistence of the epidemic. At last, we further study the threshold dynamics of a stochastic SIRS model with saturated or bilinear incidence by a similar method and carry out some numerical simulations to demonstrate our theoretical results. Comparing with the method given by Zhou and Zhang (Physica A 446:204-216, 2016), we find that the method used in this paper is simple and effective.
\end{abstract}

Keywords: Threshold dynamics; Persistence in mean; Extinction; Lévy jumps

\section{Introduction}

In recent years a large number of mathematical models based on the mechanism of disease transmission have been formulated to help us better understand how the disease spreads in the future because explicit elements of biology and behavior are included in the models [2-4]. In reality, epidemic models are inevitably affected by random environmental fluctuations, which play an important role in the study of transmission dynamics of infectious diseases [5-7]. To improve the understanding of the mechanism of disease transmission, many scholars have introduced white noise in deterministic models [8-13]. For example, Liu et al. [14] considered a stochastic SIRS epidemic model with standard incidence and established sufficient conditions for the existence of ergodic stationary distribution of the model. Fan et al. [15] established a class of SIR epidemic models with generalized nonlinear incidence rate and gave some sufficient conditions guaranteeing the extinction and persistence of the epidemic disease. Rifhat et al. [16] studied the dynamics of a class of periodic stochastic SIS epidemic models with general nonlinear incidence and gave sufficient conditions for the existence of a stochastic nontrivial periodic solution. Cai et al. [17] proposed a stochastic SIRS epidemic model with nonlinear incidence rate and presented a stochastic threshold that determines the outcome of the disease.

(c) The Author(s) 2020. This article is licensed under a Creative Commons Attribution 4.0 International License, which permits use sharing, adaptation, distribution and reproduction in any medium or format, as long as you give appropriate credit to the original author(s) and the source, provide a link to the Creative Commons licence, and indicate if changes were made. The images or other third party material in this article are included in the article's Creative Commons licence, unless indicated otherwise in a credit line to the material. If material is not included in the article's Creative Commons licence and your intended use is not permitted by statutory regulation or exceeds the permitted use, you will need to obtain permission directly from the copyright holder. To view a copy of this licence, visit http://creativecommons.org/licenses/by/4.0/. 
Let $S(t), I(t)$, and $V(t)$ denote the numbers of individuals that are susceptible to infection, of individuals that are infective, and of individuals that are immune to infection as a result of vaccination. Zhao and Jiang [18] proposed and studied the following SIVS model:

$$
\left\{\begin{array}{l}
d S=[(1-q) \Lambda-(\mu+p) S-\beta S I+\kappa I+\varepsilon V] d t+\sigma_{1} S d B_{1}(t) \\
d I=[\beta S I-(\mu+\kappa+\delta) I] d t+\sigma_{2} I d B_{2}(t) \\
d V=[q \Lambda+p S-(\mu+\varepsilon) V] d t+\sigma_{3} V d B_{3}(t)
\end{array}\right.
$$

where $\Lambda$ is the constant input of new individuals into the population per unit time, $q$ is the fraction of vaccinated for newborns, $\mu$ is the natural death rate, $p$ is the proportional coefficient of vaccinated for the susceptible, $\kappa$ is the recovery rate of infectious individuals, $\varepsilon$ is the rate of losing the immunity of vaccinated individuals, $\delta$ is the disease-caused death rate of infectious individuals, $\beta$ is the transmission coefficient between compartments $S$ and $I, B_{i}(t)(i=1,2,3)$ are standard Brownian motions defined on a complete probability space $\left(\Omega, \mathscr{F},\{\mathscr{F}\}_{t \geq 0}, \mathcal{P}\right)$ with filtration $\{\mathscr{F}\}_{t \geq 0}$ satisfying the usual conditions, and $\sigma_{i}^{2} \geq 0$ $(i=1,2,3)$ are the intensities of environmental white noise. Zhao and Jiang [18] proved that, under the condition $\mu>\frac{\sigma_{1}^{2} \vee \sigma_{2}^{2} \vee \sigma_{3}^{2}}{2}$,

- if $R_{0}^{S}<1$, then $I(t) \rightarrow 0$, that is, the disease is extinct;

- if $R_{0}^{S}>1$, then $\frac{1}{t} \int_{0}^{t} I(s) d s \rightarrow \frac{\mu(\mu+\varepsilon+p)(\mu+\kappa+\delta)}{\beta(\mu+\delta)(\mu+\varepsilon)}\left(R_{0}^{S}-1\right)$, that is, the disease is persistent in mean,

where $R_{0}^{S}=\frac{\beta \Lambda(\mu(1-q)+\varepsilon)}{\mu(\mu+\kappa+\delta)(\mu+\varepsilon+p)}-\frac{\sigma_{2}^{2}}{2(\mu+\kappa+\delta)} \equiv R_{0}-\frac{\sigma_{2}^{2}}{2(\mu+\kappa+\delta)}$, that is, $R_{0}^{S}$ is the stochastic threshold of model (1), which can completely determine the extinction and persistence of the disease, and $R_{0}$ is the threshold of the corresponding deterministic model of (1). It is worth noting that the incidence rate in (1) is bilinear, whereas some researchers point out that the disease transmission process can have a nonlinear incidence rate $[19,20]$. Capasso and Serio [19] introduced a saturated incidence rate $\beta S I /(1+a I)$ into the epidemic model, where $a>0$ is the infection force of disease.

Meanwhile, epidemic systems may suffer sudden environmental shocks, such as medical negligence, floods, toxic pollutants, and so on. These discontinuous environmental factors break the continuity of the solution and seriously affect the transmission process of the disease, but it cannot be described by white noise. Therefore some researchers turn to use the non-Gaussian Lévy noise to model these discontinuous abrupt environmental shocks in nature [21-26]. Particularly, Zhang and Wang [27] introduced Lévy jumps into a stochastic SIR model and discussed the asymptotic behavior around the equilibriums of the deterministic model. Based on this model, Zhou and Zhang [1] further investigated the effect of Lévy jumps on the dynamics of a stochastic SIR epidemic model and found a threshold, which was not accurately given in [27].

Motivated by our discussion, in this paper, we devote our main attention to investigating a stochastic SIVS model with saturated incidence driven by Lévy noise as follows:

$$
\left\{\begin{aligned}
d S= & {\left[(1-q) \Lambda-(\mu+p) S-\frac{\beta S I}{1+a I}+\kappa I+\varepsilon V\right] d t+\sigma_{1} S d B_{1}(t) } \\
& +\int_{\mathbb{Y}} \gamma_{1}(u) S\left(t^{-}\right) \widetilde{N}(d t, d u), \\
d I= & {\left[\frac{\beta S I}{1+a I}-(\mu+\kappa+\delta) I\right] d t+\sigma_{2} I d B_{2}(t)+\int_{\mathbb{Y}} \gamma_{2}(u) I\left(t^{-}\right) \tilde{N}(d t, d u), } \\
d V= & {[q \Lambda+p S-(\mu+\varepsilon) V] d t+\sigma_{3} V d B_{3}(t)+\int_{\mathbb{Y}} \gamma_{3}(u) V\left(t^{-}\right) \widetilde{N}(d t, d u), }
\end{aligned}\right.
$$


where $S\left(t^{-}\right), I\left(t^{-}\right)$, and $V\left(t^{-}\right)$are the left limits of $S(t), I(t)$, and $V(t)$, respectively, $\widetilde{N}(d t, d u):=N(d t, d u)-\lambda(d u) d t$ is a compensated Poisson process, $N$ is the Poisson counting measure with characteristic measure $\lambda$ on a measurable subset $\mathbb{Y}$ of $(0, \infty)$ with $\lambda(\mathbb{Y})<\infty$ and $\gamma_{i}(u)>-1(i=1,2,3)$. The biological meaning of all parameters in (2) is the same as in system (1).

In the deterministic epidemic model, the threshold is an interesting and important research topic and has been well solved. However, under the influence of white noise and Lévy noise, there are few studies on the threshold dynamics of the stochastic epidemic model. On the other hand, researches on such a problem in the literature often need to limit the noise intensity, which leads to a great limitation of the obtained stochastic threshold. Therefore the main purpose of this paper is finding a new method to study the threshold behavior of a stochastic SIVS model (2) and extension of this method to other models. Comparing with the results given by Zhou and Zhang [1], we find our method to be simple and effective. This paper is organized as follows. In Sect. 2, we show the existence and uniqueness of a positive solution of model (2). In Sect. 3, under some conditions, we give the stochastic threshold of model (2) that can completely determine extinction and persistence of the disease. Furthermore, we discuss the threshold of a stochastic SIRS model with Lévy jumps by the same method in Sect. 4.

\section{Existence and uniqueness of a positive solution of model (2)}

To study the long asymptotic behavior of model (2), we first need to investigate the global existence of a positive solution. To this end, we give the following two technical assumptions.

Assumption 1 For each $m>0$, there exists $L_{m}>0$ such that

$$
\int_{\mathbb{Y}}\left|H_{i}(x, u)-H_{i}(y, u)\right|^{2} \lambda(d u) \leq L_{m}|x-y|^{2} \quad \text { with }|x| \vee|y| \leq m,
$$

where $H_{i}(x, u)=\gamma_{i}(u) x\left(t^{-}\right), i=1,2,3$.

Assumption 2 There exist positive constants $K_{i}(i=1,2,3)$ such that

$$
\left|\ln \left(1+\gamma_{i}(u)\right)\right| \leq K_{i} \quad \text { for } \gamma_{i}>-1
$$

Theorem 1 Under Assumptions 1 and 2 , for any initial value $(S(0), I(0), V(0)) \in \mathbb{R}_{+}^{3}$, system (2) has a unique global solution $(S(t), I(t), V(t)) \in \mathbb{R}_{+}^{3}$ for all $t \geq 0$ almost surely.

Proof By Assumption 1, for any given initial value $(S(0), I(0), V(0)) \in \mathbb{R}_{+}^{3}$, there is a unique local solution $(S(t), I(t), V(t))$ on $t \in\left[0, \tau_{e}\right)$, where $\tau_{e}$ is the explosion time. To show that this solution is global, we need to show that $\tau_{e}=\infty$ a.s. Adopting the approach similar to that in $[1,26]$, we define the stopping time by

$$
\tau_{n}=\inf \left\{t \in\left[0, \tau_{e}\right): \min \{S(t), I(t), V(t)\} \leq \frac{1}{n} \text { or } \max \{S(t), I(t), V(t)\} \geq n\right\},
$$

where $\inf \emptyset=\infty$. Set $\tau_{\infty}=\lim _{n \rightarrow \infty} \tau_{n}$; then $\tau_{\infty} \leq \tau_{e}$ a.s. If we can show that $\tau_{\infty}=\infty$, then $\tau_{e}=\infty$ and $(S(t), I(t), V(t)) \in \mathbb{R}_{+}^{3}$ a.s. for all $t>0$. If this statement is false, then there exist 
constants $T>0$ and $\epsilon \in(0,1)$ such that $\mathcal{P}\left\{\tau_{\infty} \leq T\right\}>\epsilon$. Hence there is an integer $n_{1} \geq n_{0}$ such that $\mathcal{P}\left\{\tau_{n} \leq T\right\} \geq \epsilon$ for all $n \geq n_{1}$. Define the function $V: \mathbb{R}_{+}^{3} \rightarrow \mathbb{R}_{+}$as follows:

$$
V(S(t), I(t), V(t))=\left(S-c-c \log \frac{S}{c}\right)+(I-1-\log I)+(V-1-\log V),
$$

where $0<c<\frac{\mu+\delta}{\beta}$. Using the generalized Itô formula, we obtain

$$
\begin{aligned}
\mathscr{L} V(S, I, V)= & \left(1-\frac{c}{S}\right)\left[(1-q) \Lambda-(\mu+p) S-\frac{\beta S I}{1+a I}+\kappa I+\varepsilon V\right] \\
& +\frac{(V-1)[q \Lambda+p S-(\mu+\varepsilon) V]}{V}+\frac{(I-1)\left[\frac{\beta S I}{1+a I}-(\mu+\kappa+\delta) I\right]}{I} \\
& +\int_{\mathbb{Y}}\left[c \gamma_{1}(u)-c \ln \left(1+\gamma_{1}(u)\right)\right] \lambda(d u)+\frac{1}{2}\left(c \sigma_{1}^{2}+\sigma_{2}^{2}+\sigma_{3}^{2}\right) \\
& +\int_{\mathbb{Y}}\left[\gamma_{2}(u)-\ln \left(1+\gamma_{2}(u)\right)+\gamma_{3}(u)-\ln \left(1+\gamma_{3}(u)\right)\right] \lambda(d u) \\
= & \Lambda+c(\mu+p)+2 \mu+\kappa+\delta+\varepsilon-\mu S-\mu V-\frac{c(1-q) \Lambda}{S} \\
& -\frac{c(\kappa I+\varepsilon V)}{S}-\frac{\beta S}{1+a I}-\frac{q \Lambda}{V}-\frac{p S}{V}-\left(\mu+\delta-\frac{c \beta}{1+a I}\right) I \\
& +\int_{\mathbb{Y}}\left[c \gamma_{1}(u)-c \ln \left(1+\gamma_{1}(u)\right)\right] \lambda(d u)+\frac{1}{2}\left(c \sigma_{1}^{2}+\sigma_{2}^{2}+\sigma_{3}^{2}\right) \\
& +\int_{\mathbb{Y}}\left[\gamma_{2}(u)-\ln \left(1+\gamma_{2}(u)\right)+\gamma_{3}(u)-\ln \left(1+\gamma_{3}(u)\right)\right] \lambda(d u) .
\end{aligned}
$$

Using the inequality $x-\ln (x+1) \geq 0$ for $x>-1$ and Assumption 2, we get

$$
\mathscr{L} V(S, I, V) \leq(\Lambda+c(\mu+p)+2 \mu+\kappa+\delta+\varepsilon)+\frac{1}{2}\left(c \sigma_{1}^{2}+\sigma_{2}^{2}+\sigma_{3}^{2}\right)+3 M:=K
$$

where $M=\max _{i=2,3}\left\{\int_{\mathbb{Y}}\left[c \gamma_{1}(u)-c \ln \left(1+\gamma_{1}(u)\right)\right] \lambda(d u), \int_{\mathbb{Y}}\left[\gamma_{i}(u)-\ln \left(1+\gamma_{i}(u)\right)\right] \lambda(d u)\right\}$. Then using a similar discussion as in [1], we obtain the desired result.

\section{The threshold of model (2)}

In model (1), there exists a stochastic threshold $R_{0}^{S}$ that completely determines the extinction and prevalence of disease. When the Lévy noise is considered in model (2), we also try to find such a threshold $R_{0}^{L}$. Now let us introduce some notations and useful lemmas:

$$
\begin{array}{ll}
M_{1}(t)=\sigma_{1} \int_{0}^{t} S(s) d B_{1}(s), & M_{2}(t)=\int_{0}^{t} \int_{\mathbb{Y}} \gamma_{1}(u) S\left(s^{-}\right) \tilde{N}(d s, d u), \\
M_{3}(t)=\sigma_{2} \int_{0}^{t} I(s) d B_{2}(s), & M_{4}(t)=\int_{0}^{t} \int_{\mathbb{Y}} \gamma_{2}(u) I\left(s^{-}\right) \tilde{N}(d s, d u), \\
M_{5}(t)=\sigma_{3} \int_{0}^{t} V(s) d B_{3}(s), \quad M_{6}(t)=\int_{0}^{t} \int_{\mathbb{Y}} \gamma_{3}(u) V\left(s^{-}\right) \tilde{N}(d s, d u), \\
\tilde{M}(t)=\int_{0}^{t} \int_{\mathbb{Y}} \ln \left(1+\gamma_{2}(u)\right) \tilde{N}(d t, d u), \quad \alpha=\frac{1}{2} \sigma_{2}^{2}+\int_{\mathbb{Y}}\left[\gamma_{2}(u)-\ln \left(1+\gamma_{2}(u)\right)\right] \lambda(d u),
\end{array}
$$




$$
R_{0}^{L}=\frac{1}{\mu+\kappa+\delta}\left(\frac{\beta \Lambda(\mu(1-q)+\varepsilon)}{\mu(\mu+\varepsilon+p)}-\alpha\right), \quad\langle f(t)\rangle=\frac{1}{t} \int_{0}^{t} f(s) d s .
$$

The following lemmas are elementary.

Lemma 1 ([28]) Let $A(t)$ and $U(t)$ be two continuous adapted increasing processes on $t \geq 0$ with $A(0)=U(0)=0$ a.s. Let $M(t)$ be a real-valued continuous local martingale with $M(0)=$ 0 a.s. Let $X_{0}$ be a nonnegative $F_{0}$-measurable random variable such that $E X_{0}<\infty$. Define $X(t)=X_{0}+A(t)-U(t)+M(t)$ for $t \geq 0$. If $X(t)$ is nonnegative, then $\lim _{t \rightarrow \infty} A(t)<\infty$ implies that $\lim _{t \rightarrow \infty} U(t)<\infty, \lim _{t \rightarrow \infty} X(t)<\infty$, and $-\infty<\lim _{t \rightarrow \infty} M(t)<\infty$ with probability one.

Lemma $2([29])$ Let $M(t), t \geq 0$, be a local martingale vanishing at time 0 . Define

$$
\rho_{M}(t):=\int_{0}^{t} \frac{d\langle M, M\rangle(s)}{(1+s)^{2}}, \quad t \geq 0,
$$

where $\langle M, M\rangle(t)$ is the Meyer angle-bracket process. Then

$$
\lim _{t \rightarrow \infty} \frac{M(t)}{t}=0 \quad \text { a.s., provided that } \limsup _{t \rightarrow \infty} \rho_{M}(t)<\infty \text { a.s. }
$$

Remark 1 ([29]) Suppose that

$$
\psi_{\text {loc }}^{2}:=\left\{\psi(t, z) \text { predictable }\left.\left|\int_{0}^{t} \int_{\mathbb{Y}}\right| \psi(s, z)\right|^{2} \lambda(d u) d s<\infty\right\}
$$

and, for $\psi \in \psi_{\text {loc }}^{2}$

$$
M(t)=\int_{0}^{t} \int_{\mathbb{Y}} \psi(s, z) \tilde{N}(d s, d u)
$$

Then, by [30, Proposition 2.4],

$$
\langle M, M\rangle(t)=\int_{0}^{t} \int_{\mathbb{Y}}|\psi(s, z)|^{2} \lambda(d u) d s
$$

Lemma $3([31])$ Let $f \in C[[0, \infty) \times \Omega,(0, \infty)]$. Suppose there exist positive constants $\delta_{0}, \delta$ such that

$$
\log f(t)=\delta t-\delta_{0} \int_{0}^{t} f(s) d s+F(t) \quad \text { a.s. }
$$

for all $t \geq 0$, where $F \in C[[0, \infty) \times \Omega,(-\infty, \infty)]$ and $\lim _{t \rightarrow \infty} \frac{F(t)}{t}=0$ a.s. Then

$$
\lim _{t \rightarrow \infty}\langle f(t)\rangle=\frac{\delta}{\delta_{0}} .
$$


Lemma 4 ([32]) Let $F, G, f, g: \mathbb{R}_{+} \rightarrow \mathbb{R}$ and $H, h: \mathbb{R}_{+} \times \mathbb{Y} \rightarrow \mathbb{R}$ be Borel-measurable bounded functions such that $H>-1$, and let $Y(t)$ satisfy the equation

$$
\begin{aligned}
d Y(t)= & {[F(t) Y(t)+f(t)] d t+[G(t) Y(t)+g(t)] d W(t) } \\
& +\int_{\mathbb{Y}}\left[Y\left(t^{-}\right) H(t, u)+h(t, u)\right] \tilde{N}(d t, d u),
\end{aligned}
$$

where $Y(0)=Y_{0}$. Then the solution can be explicitly expressed as

$$
\begin{aligned}
Y(t)= & \Phi(t)\left(Y_{0}+\int_{0}^{t} \Phi^{-1}(s)\left[\left(f(s)-G(s) g(s)-\int_{\mathbb{Y}} \frac{H(s, u) h(s, u)}{1+H(s, u)} \lambda(d u)\right) d s\right.\right. \\
& \left.\left.+g(s) d W(s)+\int_{\mathbb{Y}} \frac{h(s, u)}{1+H(s, u)} \tilde{N}(d s, d u)\right]\right),
\end{aligned}
$$

where

$$
\begin{aligned}
\Phi(t)= & \exp \left[\int_{0}^{t}\left(F(s)-\frac{1}{2} G^{2}(s)+\int_{\mathbb{Y}}[\ln (1+H(s, u))-H(s, u)] \lambda(d u)\right) d s\right. \\
& \left.+\int_{0}^{t} G(s) d W(s)+\int_{0}^{t} \int_{\mathbb{Y}} \ln (1+H(s, u)) \tilde{N}(d s, d u)\right]
\end{aligned}
$$

is the fundamental solution of the corresponding homogeneous linear equation

$$
d Z(t)=F(t) Z(t) d t+G(t) Z(t) d W(t)+\int_{\mathbb{Y}} Z\left(t^{-}\right) H(t, u) \tilde{N}(d t, d u) .
$$

Lemma 5 Let the conditions of Theorem 1 hold. Assume further that there exists a constant $L>0$ such that

$$
\int_{0}^{t} \int_{\mathbb{Y}} \gamma_{i}^{2}(u) \lambda(d u) d s \leq L t, \quad i=1,2,3 .
$$

Then the solution $(S(t), I(t), V(t))$ of system (2) with initial value $(S(0), I(0), V(0)) \in \mathbb{R}_{+}^{3}$ has the following properties:

$$
\limsup _{t \rightarrow \infty}[S(t)+I(t)+V(t)]<\infty \quad \text { a.s. }
$$

and $\lim _{t \rightarrow \infty} \frac{M_{j}(t)}{t}=0(j=1,2, \ldots, 6)$ a.s.

Proof From (2) we can obtain

$$
\begin{aligned}
d(S+I+V)= & {[\Lambda-\mu S-(\mu+\delta) I-\mu V] d t+\sigma_{1} S d B_{1}(t)+\sigma_{2} I d B_{2}(t)+\sigma_{3} V d B_{3}(t) } \\
& +\int_{\mathbb{Y}}\left[\gamma_{1}(u) S\left(t^{-}\right)+\gamma_{2}(u) I\left(t^{-}\right)+\gamma_{3}(u) V\left(t^{-}\right)\right] \widetilde{N}(d t, d u) .
\end{aligned}
$$

By Lemma 4 the solution of Eq. (4) has the following form:

$$
\begin{aligned}
S(t) & +I(t)+V(t) \\
& =\frac{\Lambda}{\mu}+\left[S(0)+I(0)+V(0)-\frac{\Lambda}{\mu}\right] e^{-\mu t}-\delta \int_{0}^{t} e^{-\mu(t-s)} I(s) d s+M(t)
\end{aligned}
$$




$$
\leq \frac{\Lambda}{\mu}+\left[S(0)+I(0)+V(0)-\frac{\Lambda}{\mu}\right] e^{-\mu t}+M(t)
$$

where

$$
\begin{aligned}
M(t)= & \sigma_{1} \int_{0}^{t} e^{-\mu(t-s)} S(s) d B_{1}(s)+\sigma_{2} \int_{0}^{t} e^{-\mu(t-s)} I(s) d B_{2}(s) \\
& +\sigma_{3} \int_{0}^{t} e^{-\mu(t-s)} V(s) d B_{3}(s)+\int_{0}^{t} \int_{\mathbb{Y}} e^{-\mu(t-s)} \gamma_{1}(u) S\left(s^{-}\right) \tilde{N}(d s, d u) \\
& +\int_{0}^{t} \int_{\mathbb{Y}} e^{-\mu(t-s)} \gamma_{2}(u) I\left(s^{-}\right) \tilde{N}(d s, d u)+\int_{0}^{t} \int_{\mathbb{Y}} e^{-\mu(t-s)} \gamma_{3}(u) V\left(s^{-}\right) \tilde{N}(d s, d u)
\end{aligned}
$$

is a continuous local martingale with $M(0)=0$.

Denote $X(t)=\frac{\Lambda}{\mu}+\left[S(0)+I(0)+V(0)-\frac{\Lambda}{\mu}\right] e^{-\mu t}+M(t), X(0)=S(0)+I(0)+V(0), A(t)=$ $\frac{\Lambda}{\mu}\left(1-e^{-\mu t}\right)$, and $U(t)=(S(0)+I(0)+V(0))\left(1-e^{-\mu t}\right)$. Then $X(t)=X(0)+A(t)-U(t)+M(t)$ and $A(t)$ and $U(t)$ are continuous adapted increasing processes on $t \geq 0$ with $A(0)=U(0)=$ 0 . Then Lemma 1 implies that $\lim _{t \rightarrow \infty} X(t)<\infty$, which leads to

$$
\limsup _{t \rightarrow \infty}[S(t)+I(t)+V(t)]<\infty \quad \text { a.s. }
$$

On the other hand, simple calculation shows that

$$
\left\langle M_{1}, M_{1}\right\rangle(t)=\sigma_{1}^{2} \int_{0}^{t} S^{2}(s) d s
$$

and thus

$$
\limsup _{t \rightarrow \infty} \rho_{1}(t)=\limsup _{t \rightarrow \infty} \int_{0}^{t} \frac{\sigma_{1}^{2} S^{2}(s) d s}{(1+s)^{2}} \leq \sigma_{1}^{2} \sup _{t \geq 0} S^{2}(t)<\infty
$$

According to Lemma $2, \lim _{t \rightarrow \infty} \frac{M_{1}}{t}=0$ a.s. Meanwhile, by Remark 1 and condition (3) we get

$$
\left\langle M_{2}, M_{2}\right\rangle(t)=\int_{0}^{t} \int_{\mathbb{Y}} \gamma_{1}^{2}(u) S^{2}(s) \lambda(d u) d s
$$

and thus

$$
\limsup _{t \rightarrow \infty} \rho_{2}(t)=\limsup _{t \rightarrow \infty} \int_{0}^{t} \int_{\mathbb{Y}} \frac{\gamma_{1}^{2}(u) S^{2}(s)}{(1+s)^{2}} \lambda(d u) d s \leq L \sup _{t \geq 0} S^{2}(t)<\infty
$$

Applying Lemma 2 again yields $\lim _{t \rightarrow \infty} \frac{M_{2}}{t}=0$ a.s. The rest of Lemma 5 can be proved similarly. The proof is complete.

We are now in position to state and prove our main results of this paper.

Theorem 2 Let the conditions of Lemma 5 hold, and let $(S(t), I(t), V(t))$ be the solution of system (2) with initial value $(S(0), I(0), V(0)) \in \mathbb{R}_{+}^{3}$. 
(I) If $R_{0}^{L}<1$, then

$$
\limsup _{t \rightarrow \infty} \frac{\ln I(t)}{t} \leq(\mu+\kappa+\delta)\left(R_{0}^{L}-1\right)<0 \quad \text { a.s. }
$$

which means that $\lim _{t \rightarrow \infty} I(t)=0$ a.s.

Moreover,

$$
\lim _{t \rightarrow \infty}\langle S(t)\rangle=\frac{(\mu(1-q)+\varepsilon) \Lambda}{\mu(\mu+\varepsilon+p)}, \quad \lim _{t \rightarrow \infty}\langle V(t)\rangle=\frac{(p+\mu q) \Lambda}{\mu(\mu+\varepsilon+p)} \quad \text { a.s. }
$$

(II) If $R_{0}^{L}>1$, then

$$
\lim _{t \rightarrow \infty}\langle I(t)\rangle=\frac{\mu+\kappa+\delta}{\mu+\delta}\left(a\left(1+\frac{\kappa}{\mu+\delta}\right)+\frac{\beta(\mu+\varepsilon)}{\mu(\mu+\varepsilon+p)}\right)^{-1}\left(R_{0}^{L}-1\right)>0 \quad \text { a.s. }
$$

Moreover,

$$
\lim _{t \rightarrow \infty}\langle S(t)\rangle=\widetilde{S}^{*}, \quad \lim _{t \rightarrow \infty}\langle V(t)\rangle=\widetilde{V}^{*} \quad \text { a.s. },
$$

where $\widetilde{S}^{*}=\frac{(\mu(1-q)+\varepsilon) \Lambda}{\mu(\mu+\varepsilon+p)}+\frac{(\mu+\varepsilon)(\mu+\delta)\left(\mu+\kappa+\delta+\alpha-\frac{\beta \Lambda(\mu(1-q)+\varepsilon)}{\mu(\mu+\varepsilon+p)}\right)}{a \mu(\mu+\varepsilon+p)(\mu+\kappa+\delta)+\beta(\mu+\varepsilon)(\mu+\delta)}$ and $\widetilde{V}^{*}=\frac{q \Lambda+p \widetilde{S}^{*}}{\mu+\varepsilon}$.

Proof From system (2) we get

$$
\begin{aligned}
d(S+ & I)+\frac{\varepsilon}{\mu+\varepsilon} d V \\
= & \left(\frac{\mu(1-q)+\varepsilon}{\mu+\varepsilon} \Lambda-(\mu+p) S-(\mu+\delta) I+\frac{\varepsilon p}{\mu+\varepsilon} S\right) d t+\sigma_{1} S d B_{1}(t) \\
& +\sigma_{2} I d B_{2}(t)+\sigma_{3} V d B_{3}(t)+\int_{\mathbb{Y}} \gamma_{1}(u) S\left(t^{-}\right) \tilde{N}(d t, d u) \\
& +\int_{\mathbb{Y}} \gamma_{2}(u) I\left(t^{-}\right) \tilde{N}(d t, d u)+\int_{\mathbb{Y}} \gamma_{3}(u) V\left(t^{-}\right) \tilde{N}(d t, d u) .
\end{aligned}
$$

Integrating this from 0 to $t$ and dividing both sides by $t$, we have

$$
\frac{\mu(1-q)+\varepsilon}{\mu+\varepsilon} \Lambda-\frac{\mu(\mu+\varepsilon+p)}{\mu+\varepsilon}\langle S(t)\rangle-(\mu+\delta)\langle I(t)\rangle=\frac{1}{t} \varphi_{1}(t)
$$

where $\varphi_{1}(t)=S(t)+I(t)-S(0)-I(0)+\frac{\varepsilon}{\mu+\varepsilon}(V(t)-V(0))-\sum_{i=1}^{4} M_{i}-\frac{\varepsilon}{\mu+\varepsilon}\left(M_{5}+M_{6}\right)$. Similarly,

$$
(1-q) \Lambda-\left(\mu+p+\frac{\beta}{a}\right)\langle S(t)\rangle+\frac{1}{a}\left\langle\frac{\beta S}{1+a I}\right\rangle+\kappa\langle I(t)\rangle+\varepsilon\langle V(t)\rangle=\frac{1}{t} \varphi_{2}(t),
$$

and

$$
q \Lambda+p\langle S(t)\rangle-(\mu+\varepsilon)\langle V(t)\rangle=\frac{1}{t} \varphi_{3}(t)
$$

where $\varphi_{2}(t)=S(t)-S(0)-M_{1}(t)-M_{2}(t), \varphi_{3}(t)=V(t)-V(0)-M_{5}(t)-M_{6}(t)$. Then by Lemma 5 we have

$$
\lim _{t \rightarrow \infty} \frac{\varphi_{1}(t)}{t}=0, \quad \lim _{t \rightarrow \infty} \frac{\varphi_{2}(t)}{t}=0, \quad \lim _{t \rightarrow \infty} \frac{\varphi_{3}(t)}{t}=0 .
$$


Applying Itô's formula to the second equation of system (2) leads to

$$
\frac{\ln I(t)-\ln I(0)}{t}=\left\langle\frac{\beta S}{1+a I}\right\rangle-(\mu+\kappa+\delta)-\alpha+\frac{\sigma_{2} B_{2}(t)}{t}+\frac{\tilde{M}}{t} .
$$

Substituting (5)-(7) into (9) yields that

$$
\begin{aligned}
\frac{\ln I(t)}{t}= & \frac{\beta \Lambda(\mu(1-q)+\varepsilon)}{\mu(\mu+\varepsilon+p)}-(\mu+\kappa+\delta)-\alpha \\
& -\left(a \kappa+(\mu+\delta)\left(a+\frac{\beta(\mu+\varepsilon)}{\mu(\mu+\varepsilon+p)}\right)\right)\langle I(t)\rangle+\frac{F_{1}(t)}{t},
\end{aligned}
$$

where $F_{1}(t)=-\left(a+\frac{\beta(\mu+\varepsilon)}{\mu(\mu+\varepsilon+p)}\right) \varphi_{1}(t)+a \varphi_{2}(t)+\frac{a \varepsilon}{\mu+\varepsilon} \varphi_{3}(t)+\ln I(0)+\sigma_{2} B_{2}(t)+\widetilde{M}$. According to Remark 1 and Assumption 2, this implies

$$
\langle\tilde{M}, \tilde{M}\rangle(t)=\int_{0}^{t} \int_{\mathbb{Y}}\left[\ln \left(1+\gamma_{2}(u)\right]^{2} \lambda(d u) d s \leq K_{2}^{2} \lambda(\mathbb{Y}) t .\right.
$$

Then by Lemma 2

$$
\lim _{t \rightarrow \infty} \frac{\tilde{M}}{t}=0 \quad \text { a.s. }
$$

On the other hand, by the large number theorem for martingales we obtain

$$
\lim _{t \rightarrow \infty} \frac{B_{2}(t)}{t}=0 \quad \text { a.s. }
$$

This, together with (8) and (11), yields

$$
\lim _{t \rightarrow \infty} \frac{F_{1}(t)}{t}=0 \quad \text { a.s. }
$$

According to (13) and $I(t)>0$, taking the limit superior of both sides of (10), we have

$$
\limsup _{t \rightarrow \infty} \frac{\ln I(t)}{t} \leq(\mu+\kappa+\delta)\left(R_{0}^{L}-1\right)<0 \quad \text { a.s. }
$$

if $R_{0}^{L}<1$. Furthermore, from (3), (7), and (8) we easily to see that

$$
\lim _{t \rightarrow \infty}\langle S(t)\rangle=\frac{(\mu(1-q)+\varepsilon) \Lambda}{\mu(\mu+\varepsilon+p)}, \quad \lim _{t \rightarrow \infty}\langle V(t)\rangle=\frac{(p+\mu q) \Lambda}{\mu(\mu+\varepsilon+p)} \quad \text { a.s. }
$$

If $R_{0}^{L}>1$, from (10), (13), and Lemma 3 we get

$$
\lim _{t \rightarrow \infty}\langle I(t)\rangle=\frac{\mu+\kappa+\delta}{\mu+\delta}\left(a\left(1+\frac{\kappa}{\mu+\delta}\right)+\frac{\beta(\mu+\varepsilon)}{\mu(\mu+\varepsilon+p)}\right)^{-1}\left(R_{0}^{L}-1\right)>0 \quad \text { a.s. }
$$

Meanwhile, from (5), (7), and (8) by simple calculation we have

$$
\lim _{t \rightarrow \infty}\langle S(t)\rangle=\widetilde{S}^{*}, \quad \lim _{t \rightarrow \infty}\langle V(t)\rangle=\widetilde{V}^{*} \quad \text { a.s. }
$$

This completes the proof of Theorem 2. 
Remark 2 From Theorem 2 it follows that the disease will go to extinct when $R_{0}^{L}<1$ and will prevail when $R_{0}^{L}>1$. Therefore the parameter $R_{0}^{L}$ is the threshold of model (2).

Particularly, if the corresponding incidence rate is bilinear with respect to susceptible and infective individuals, then the stochastic SIVS model with jumps has the form

$$
\left\{\begin{aligned}
d S= & {[(1-q) \Lambda-(\mu+p) S-\beta S I+\kappa I+\varepsilon V] d t+\sigma_{1} S d B_{1}(t) } \\
& +\int_{\mathbb{Y}} \gamma_{1}(u) S\left(t^{-}\right) \tilde{N}(d t, d u), \\
d I= & {[\beta S I-(\mu+\kappa+\delta) I] d t+\sigma_{2} I d B_{2}(t)+\int_{\mathbb{Y}} \gamma_{2}(u) I\left(t^{-}\right) \tilde{N}(d t, d u), } \\
d V= & {[q \Lambda+p S-(\mu+\varepsilon) V] d t+\sigma_{3} V d B_{3}(t)+\int_{\mathbb{Y}} \gamma_{3}(u) V\left(t^{-}\right) \tilde{N}(d t, d u) . }
\end{aligned}\right.
$$

By the method used in Theorem 2 we have the following conclusions.

Corollary 1 Let the conditions of Lemma 5 hold, and let $(S(t), I(t), V(t))$ be the solution of system (14) with initial value $(S(0), I(0), V(0)) \in \mathbb{R}_{+}^{3}$.

(I) If $R_{0}^{L}<1$, then

$$
\limsup _{t \rightarrow \infty} \frac{\ln I(t)}{t} \leq(\mu+\kappa+\delta)\left(R_{0}^{L}-1\right)<0 \quad \text { a.s. }
$$

that is, $\lim _{t \rightarrow \infty} I(t)=0$ a.s.

Moreover,

$$
\lim _{t \rightarrow \infty}\langle S(t)\rangle=\frac{(\mu(1-q)+\varepsilon) \Lambda}{\mu(\mu+\varepsilon+p)}, \quad \lim _{t \rightarrow \infty}\langle V(t)\rangle=\frac{(p+\mu q) \Lambda}{\mu(\mu+\varepsilon+p)} \quad \text { a.s. }
$$

(II) If $R_{0}^{L}>1$, then

$$
\lim _{t \rightarrow \infty}\langle I(t)\rangle=\frac{\mu(\mu+\kappa+\delta)(\mu+\varepsilon+p)}{\beta(\mu+\delta)(\mu+\varepsilon)}\left(R_{0}^{L}-1\right)>0 \quad \text { a.s. }
$$

Moreover,

$$
\lim _{t \rightarrow \infty}\langle S(t)\rangle=\frac{\mu+\kappa+\delta+\alpha}{\beta}, \quad \lim _{t \rightarrow \infty}\langle V(t)\rangle=\frac{q \Lambda+\frac{p}{\beta}(\mu+\kappa+\delta+\alpha)}{\mu+\varepsilon} \text { a.s. }
$$

Remark 3 We easily find that the parameter $R_{0}^{L}$ is also the threshold of model (14). Obviously, $R_{0}^{L}<R_{0}^{S}<R_{0}$, that is, a Lévy noise is able to suppress the outbreak of the disease. The model considered in [18] is a particular case of model (14) $\left(\gamma_{i}(u)=0, i=1,2,3\right)$. In comparison with [18], our Corollary 1 improves and extends the related results.

Example 1 Let $(S, I, V)$ be the solution of model (14) with $(S(0), I(0), V(0))=(0.8,0.1,2)$. $\Lambda=0.7, q=0.7, \mu=0.21, p=0.5, \beta=0.75, \kappa=0.3, \varepsilon=0.2, \delta=0.2, \sigma_{1}=0.01, \sigma_{2}=0.05$, $\sigma_{3}=0.01, \gamma_{1}=0.08, \gamma_{2}=0.2, \gamma_{3}=0.08, \mathbb{Y}=(0, \infty), \lambda(\mathbb{Y})=1$. We use these parameters to simulate the related results.

Through simple calculation, we have $R_{0}^{L}=0.991<1$. Then the disease will go to extinction by Corollary 1; see Fig. 1. However, for the corresponding deterministic model and 

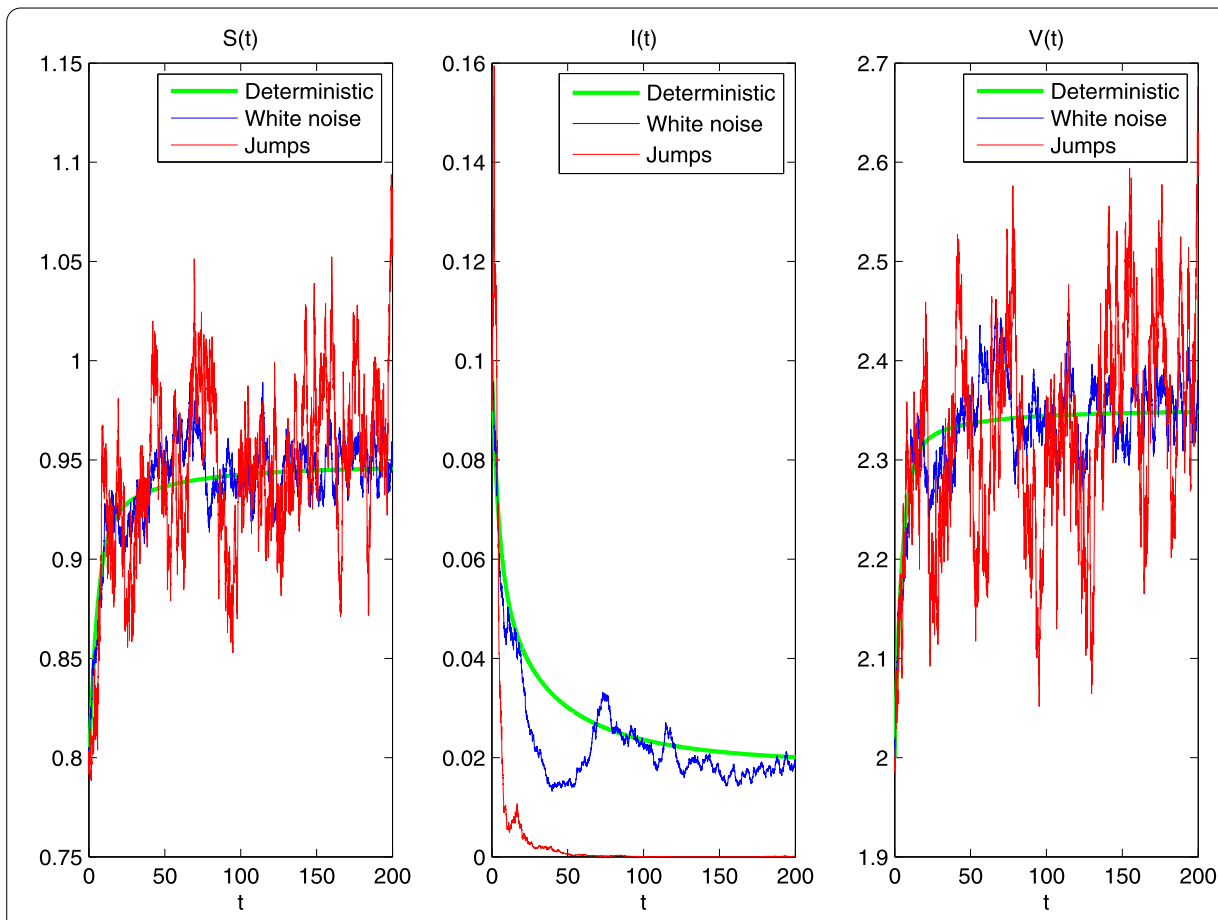

Figure 1 The trajectories of stochastic model (14) with jumps $\left(R_{0}^{L}<1\right)$, stochastic model (1) with white noise $\left(R_{0}^{S}>1\right)$, and corresponding deterministic model $\left(R_{0}>1\right)$

stochastic model with white noise, the disease is persistent since $R_{0}=\frac{\beta \Lambda(\mu(1-q)+\varepsilon)}{\mu(\mu+\kappa+\delta)(\mu+\varepsilon+p)}=$ $1.0176>1$ and $R_{0}^{S}=R_{0}-\frac{\sigma_{2}^{2}}{2(\mu+\kappa+\delta)}=1.0159>1$, respectively; see Fig. 1 .

In Fig. 2, we choose $\gamma_{2}=0.05$, and other parameters remain unchanged. Note that $R_{0}^{L}=$ $1.0142>1$, so that by Corollary 1 the disease will prevail. Moreover,

$$
\begin{aligned}
& \lim _{t \rightarrow \infty}\langle I(t)\rangle=\frac{\mu(\mu+\kappa+\delta)(\mu+\varepsilon+p)}{\beta(\mu+\delta)(\mu+\varepsilon)}\left(R_{0}^{L}-1\right)=0.0153, \\
& \lim _{t \rightarrow \infty}\langle S(t)\rangle=\frac{\mu+\kappa+\delta+\alpha}{\beta}=0.9499, \quad \text { and } \\
& \lim _{t \rightarrow \infty}\langle V(t)\rangle=\frac{q \Lambda+\frac{p}{\beta}(\mu+\kappa+\delta+\alpha)}{\mu+\varepsilon}=2.3536 .
\end{aligned}
$$

Comparing Figs. 1 and 2, we can see that a Lévy noise can suppress the outbreak of the disease.

\section{Extensions}

In this section, using the same method as before, we investigate the threshold of stochastic SIRS model with saturated or bilinear incidence driven by Lévy noise. Zhang and Wang [27] considered a stochastic SIR model with jumps and the corresponding incidence rate, which is bilinear with respect to the numbers of susceptible and infective individuals. If we consider the transmission of the disease governed by the saturated incidence rate $\beta S /(1+$ $a I)$ and the recovered individuals lose immunity and return to the susceptible class at the 

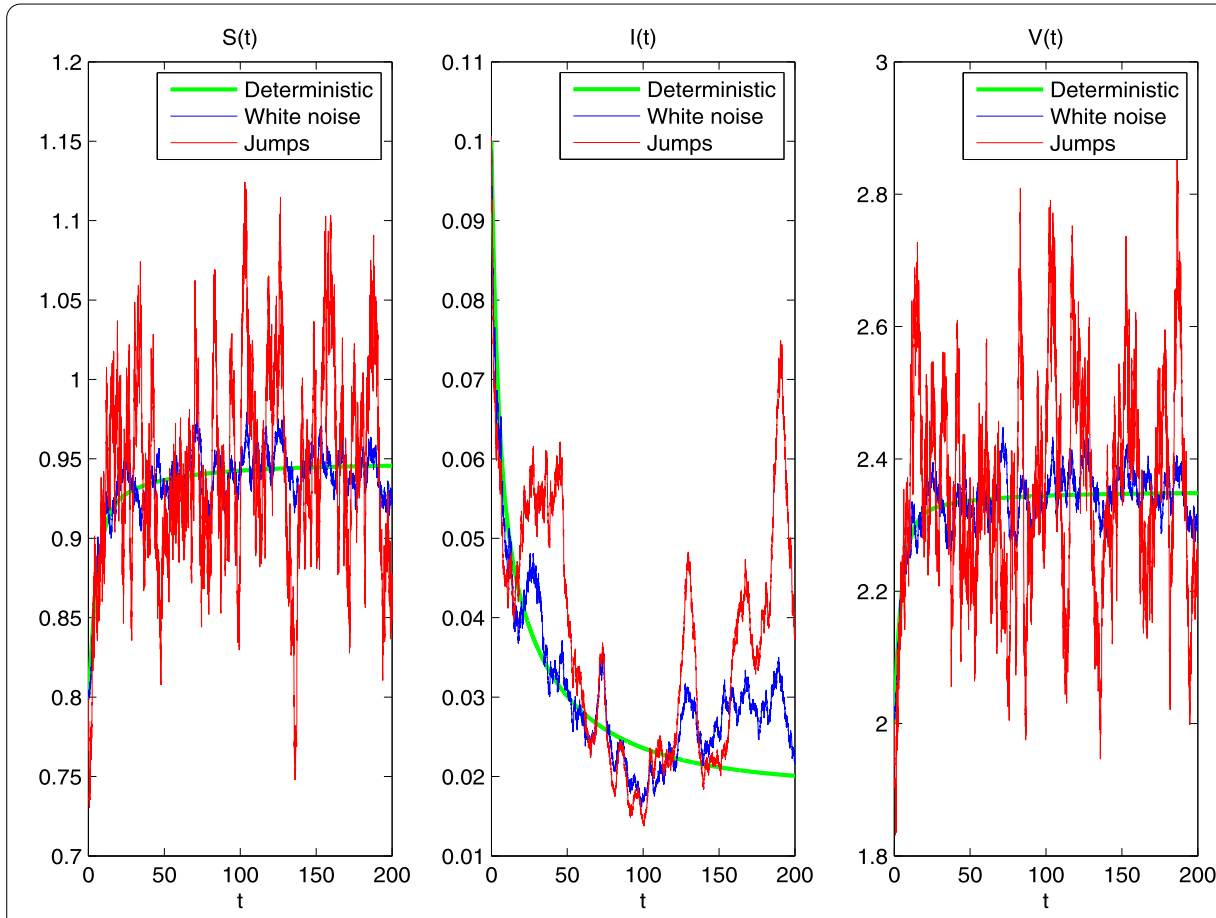

Figure 2 The trajectories of stochastic model (14) with jumps $\left(R_{0}^{L}>1\right)$, stochastic model (1) with white noise $\left(R_{0}^{S}>1\right)$, and corresponding deterministic model $\left(R_{0}>1\right)$

rate $\varepsilon$, then the model in [27] takes the following form:

$$
\left\{\begin{array}{l}
d S=\left(\Lambda-\mu S-\frac{\beta S I}{1+a I}+\varepsilon R\right) d t+\sigma_{1} S d B_{1}(t)+\int_{\mathbb{Y}} \gamma_{1}(u) S\left(t^{-}\right) \tilde{N}(d t, d u), \\
d I=\left(\frac{\beta S I}{1+a I}-(\mu+\kappa+\delta) I\right) d t+\sigma_{2} I d B_{2}(t)+\int_{\mathbb{Y}} \gamma_{2}(u) I\left(t^{-}\right) \tilde{N}(d t, d u), \\
d R=(\kappa I-(\mu+\varepsilon) R) d t+\sigma_{3} R d B_{3}(t)+\int_{\mathbb{Y}} \gamma_{3}(u) R\left(t^{-}\right) \widetilde{N}(d t, d u) .
\end{array}\right.
$$

By using a similar method in Theorem 1 we can prove the existence of a unique positive solution of model (15), so we omit the proof.

Let

$$
R_{0}^{\mathcal{L}}=\frac{1}{\mu+\kappa+\delta}\left(\frac{\beta \Lambda}{\mu}-\alpha\right)
$$

where $\alpha=\frac{1}{2} \sigma_{2}^{2}+\int_{\mathbb{Y}}\left[\gamma_{2}(u)-\ln \left(1+\gamma_{2}(u)\right)\right] \lambda(d u)$.

From system (15) we obtain

$$
\begin{aligned}
& \kappa\langle I(t)\rangle-(\mu+\varepsilon)\langle R(t)\rangle=\frac{1}{t} \varphi_{4}(t), \\
& \Lambda-\left(\mu+\frac{\beta}{a}\right)\langle S(t)\rangle+\frac{1}{a}\left\langle\frac{\beta S}{1+a I}\right\rangle+\varepsilon\langle R(t)\rangle=\frac{1}{t} \varphi_{5}(t),
\end{aligned}
$$

and

$$
\Lambda-\mu\langle S(t)\rangle-\frac{(\mu+\varepsilon)(\mu+\kappa+\delta)-\varepsilon \kappa}{\mu+\varepsilon}\langle I(t)\rangle=\frac{1}{t} \varphi_{6}(t),
$$


where $\varphi_{4}(t)=R(t)-R(0)-M_{5}(t)-M_{6}(t), \varphi_{5}(t)=S(t)-S(0)-M_{1}(t)-M_{2}(t)$, and $\varphi_{6}(t)=$ $S(t)+I(t)-S(0)-I(0)+\frac{\varepsilon}{\mu+\varepsilon}(R(t)-R(0))-\sum_{i=1}^{4} M_{i}-\frac{\varepsilon}{\mu+\varepsilon}\left(M_{5}+M_{6}\right)$. Substituting (16) $-(18)$ into (9), we have

$$
\frac{\ln I(t)}{t}=\frac{\beta \Lambda}{\mu}-(\mu+\kappa+\delta)-\alpha-\frac{(a \mu+\beta)(\mu+\varepsilon)(\mu+\kappa+\delta)-\beta \varepsilon \kappa}{\mu(\mu+\varepsilon)}\langle I(t)\rangle-\frac{F_{2}(t)}{t},
$$

where

$$
F_{2}(t)=\frac{a \varepsilon}{\mu+\varepsilon} \varphi_{4}(t)+a \varphi_{5}(t)-\frac{a \mu+\beta}{\mu} \varphi_{6}(t)+\ln I(0)+\sigma_{2} B_{2}(t)+\tilde{M} .
$$

Based on (19), by a similar discussion in Sect. 3, we get the following results.

Theorem 3 Let the conditions of Lemma 5 hold, and let $(S(t), I(t), R(t))$ be the solution of system (15) with initial value $(S(0), I(0), R(0)) \in \mathbb{R}_{+}^{3}$.

(I) If $R_{0}^{\mathcal{L}}<1$, then

$$
\limsup _{t \rightarrow \infty} \frac{\ln I(t)}{t} \leq(\mu+\kappa+\delta)\left(R_{0}^{\mathcal{L}}-1\right)<0 \quad \text { a.s. }
$$

that is, $\lim _{t \rightarrow \infty} I(t)=0$ a.s.

Moreover,

$$
\lim _{t \rightarrow \infty}\langle S(t)\rangle=\frac{\Lambda}{\mu}, \quad \lim _{t \rightarrow \infty}\langle R(t)\rangle=0 \quad \text { a.s. }
$$

(II) If $R_{0}^{\mathcal{L}}>1$, then

$$
\lim _{t \rightarrow \infty}\langle I(t)\rangle=\frac{\mu(\mu+\varepsilon)(\mu+\kappa+\delta)}{(a \mu+\beta)(\mu+\varepsilon)(\mu+\kappa+\delta)-\beta \varepsilon \kappa}\left(R_{0}^{\mathcal{L}}-1\right)>0 \quad \text { a.s. }
$$

Moreover,

$$
\begin{aligned}
& \lim _{t \rightarrow \infty}\langle S(t)\rangle=\frac{\Lambda}{\mu}-\frac{[(\mu+\varepsilon)(\mu+\kappa+\delta)-\varepsilon \kappa](\mu+\kappa+\delta)}{(a \mu+\beta)(\mu+\varepsilon)(\mu+\kappa+\delta)-\beta \varepsilon \kappa}\left(R_{0}^{\mathcal{L}}-1\right), \\
& \lim _{t \rightarrow \infty}\langle R(t)\rangle=\frac{\kappa \lim _{t \rightarrow \infty}\langle I(t)\rangle}{\mu+\varepsilon} \quad \text { a.s. }
\end{aligned}
$$

Theorem 3 implies that the parameter $R_{0}^{\mathcal{L}}$ is the threshold of model (15). As a particular case, we consider the bilinear incidence rate. Then the corresponding stochastic SIRS model with jumps has the following form:

$$
\left\{\begin{array}{l}
d S=(\Lambda-\mu S-\beta S I+\varepsilon R) d t+\sigma_{1} S d B_{1}(t)+\int_{\mathbb{Y}} \gamma_{1}(u) S\left(t^{-}\right) \tilde{N}(d t, d u) \\
d I=(\beta S I-(\mu+\kappa+\delta) I) d t+\sigma_{2} I d B_{2}(t)+\int_{\mathbb{Y}} \gamma_{2}(u) I\left(t^{-}\right) \tilde{N}(d t, d u) \\
d R=(\kappa I-(\mu+\varepsilon) R) d t+\sigma_{3} R d B_{3}(t)+\int_{\mathbb{Y}} \gamma_{3}(u) R\left(t^{-}\right) \tilde{N}(d t, d u)
\end{array}\right.
$$

By Theorem 3 the following results are obvious.

Corollary 2 Let the conditions of Lemma 5 hold, and let $(S(t), I(t), R(t))$ be the solution of system (20) with initial value $(S(0), I(0), R(0)) \in \mathbb{R}_{+}^{3}$. 
(I) If $R_{0}^{\mathcal{L}}<1$, then

$$
\limsup _{t \rightarrow \infty} \frac{\ln I(t)}{t} \leq(\mu+\kappa+\delta)\left(R_{0}^{\mathcal{L}}-1\right)<0 \quad \text { a.s. }
$$

that is, $\lim _{t \rightarrow \infty} I(t)=0$ a.s.

Moreover,

$$
\lim _{t \rightarrow \infty}\langle S(t)\rangle=\frac{\Lambda}{\mu}, \quad \lim _{t \rightarrow \infty}\langle R(t)\rangle=0 \quad \text { a.s. }
$$

(II) If $R_{0}^{\mathcal{L}}>1$, then

$$
\begin{aligned}
& \lim _{t \rightarrow \infty}\langle I(t)\rangle=\frac{\mu(\mu+\kappa+\delta)(\mu+\varepsilon)}{\beta(\mu+\kappa+\delta)(\mu+\varepsilon)-\beta \varepsilon \kappa}\left(R_{0}^{\mathcal{L}}-1\right)>0 \quad \text { a.s. } \\
& \lim _{t \rightarrow \infty}\langle S(t)\rangle=\frac{\mu+\kappa+\delta+\alpha}{\beta}, \quad \lim _{t \rightarrow \infty}\langle R(t)\rangle=\frac{\kappa \lim _{t \rightarrow \infty}\langle I(t)\rangle}{\mu+\varepsilon} \quad \text { a.s. }
\end{aligned}
$$

Remark 4 In model (20), if we take $\varepsilon=0$, then it becomes a stochastic SIR model with jumps, which has been investigated by Zhou and Zhang [1]. Under the assumption that the noise is small enough, that is, for some $p>1$,

$$
\mu-\frac{p-1}{2} \sigma^{2}-\frac{p}{2} \theta>0
$$

where $\theta=\int_{\mathbb{Y}}\left[\left(1+\gamma_{1}(u) \vee \gamma_{2}(u) \vee \gamma_{3}(u)\right)^{p}-1-\left(\gamma_{1}(u) \wedge \gamma_{2}(u) \wedge \gamma_{3}(u)\right)\right] \lambda(d u)$ and $\sigma^{2}=\sigma_{1}^{2} \vee$ $\sigma_{2}^{2} \vee \sigma_{3}^{2}$, they give the threshold $R_{0}^{\mathcal{L}}=\frac{1}{\mu+\kappa+\delta}\left(\beta \frac{\Lambda}{\mu}-\alpha\right)$. However, by Corollary 2 we easily to see that we need no assumption that the noise is small enough. So the related results are improved. In comparison with the method given by Zhou and Zhang [1], our method is simple and effective by use of the nonnegative semimartingale convergence theorem.

Example 2 Let $(S, I, R)$ be the solution of model (20) with $(S(0), I(0), R(0))=(0.8,0.1,2)$, $\Lambda=0.6, \mu=0.2, \beta=0.2, \kappa=0.2, \delta=0.05, \varepsilon=0, \sigma_{1}=0.01, \sigma_{2}=0.2, \sigma_{3}=0.1, \gamma_{1}=0.01$, $\gamma_{2}=0.42, \gamma_{3}=0.01, \mathbb{Y}=(0, \infty), \lambda(\mathbb{Y})=1$.
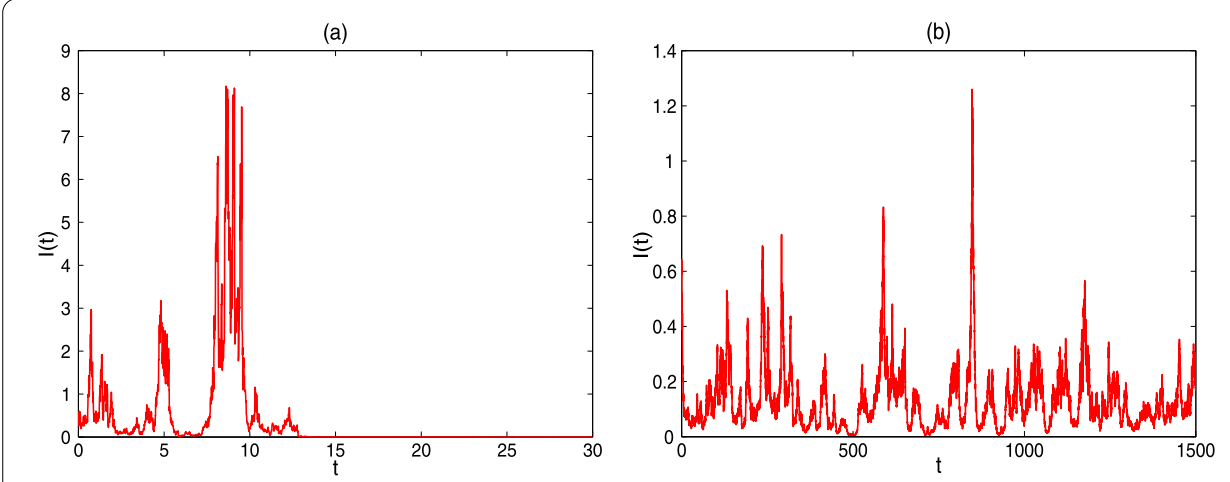

Figure 3 The trajectories of $/(t)$ for model (20) with (a) $\gamma_{2}=0.42$, (b) $\gamma_{2}=0.2$, and other parameter values as in Example 2 
When $\varepsilon=0$, model (20) becomes a stochastic SIR model with jumps. Obviously, there does not exist a constant $p>1$ such that $\mu-\frac{p-1}{2} \sigma^{2}-\frac{p}{2} \theta>0$, which means that we cannot determine whether the disease is extinct or not by the related results given in [1]. We computed that $R_{0}^{\mathcal{L}}=\frac{1}{\mu+\kappa+\delta}\left(\frac{\beta \Lambda}{\mu}-\alpha\right)=0.9704<1$. It follows from Corollary 2 that the disease goes extinct; see Fig. 3(a). When $\gamma_{2}=0.2$ and the other parameters remain unchanged, we obtain $R_{0}^{\mathcal{L}}=1.0804>1$, implying that the disease will prevail; see Fig. 3(b). Therefore Corollary 2 improves the related results in [1].

Acknowledgements

The authors thank the editor and referees for their careful reading and valuable comments.

Funding

Not applicable.

Availability of data and materials

Not applicable.

Competing interests

The authors declare that they have no competing interests.

Authors' contributions

Both authors contributed equally to this work. Both authors read and approved the final manuscript.

\section{Author details}

'School of Economics, Zhengzhou University of Aeronautics, Zhengzhou, China. ${ }^{2}$ School of Management Engineering, Zhengzhou University of Aeronautics, Zhengzhou, China.

\section{Publisher's Note}

Springer Nature remains neutral with regard to jurisdictional claims in published maps and institutional affiliations.

Received: 6 September 2019 Accepted: 25 May 2020 Published online: 09 June 2020

\section{References}

1. Zhou, Y., Zhang, W.: Threshold of a stochastic SIR epidemic model with Lévy jumps. Physica A 446, 204-216 (2016)

2. Kermack, W., McKendrick, A.: Contributions to the mathematical theory of epidemics (Part I). Proc. R. Soc. Lond. Ser. A, Math. Phys. Sci. 115, 700-721 (1927)

3. Tchuenche, J.M., Nwagwo, A., Levins, R.: Global behaviour of an SIR epidemic model with time delay. Math. Methods Appl. Sci. 30, 733-749 (2007)

4. Zhang, T., Teng, Z:: Permanence and extinction for a nonautonomous SIRS epidemic model with time delay. Appl. Math. Model. 33, 1058-1071 (2009)

5. Melbourne, B.A., Hastings, A.: Extinction risk depends strongly on factors contributing to stochasticity. Nature 45, 100-103 (2008)

6. Spagnolo, B., Valenti, D., Fiasconaro, A.: Noise in ecosystems: a short review. Math. Biosci. 1, 185-211 (2004)

7. May, R.: Stability and Complexity in Model Ecosystems. Princeton University Press, New Jersey (1973)

8. Li, D., Liu, S., Cui, J.: Threshold dynamics and ergodicity of an SIRS epidemic model with Markovian switching. J. Differ. Equ. 263, 873-8915 (2017)

9. Zhao, Y., Jiang, D., O'Regan, D.: The extinction and persistence of the stochastic SIS epidemic model with vaccination. Physica A 392, 4916-4927 (2013)

10. Gray, A., Greenhalgh, D., Hu, L., Mao, X., Pan, J.: A stochastic differential equation SIS epidemic model. SIAM J. Appl. Math. 71, 876-902 (2011)

11. Zhang, S., Meng, X., Feng, T., Zhang, T.: Dynamics analysis and numerical simulations of a stochastic non-autonomous predator-prey system with impulsive effects. Nonlinear Anal. Hybrid Syst. 26, 19-37 (2017)

12. Qi, H., Liu, L., Meng, X.: Dynamics of a nonautonomous stochastic SIS epidemic model with double epidemic hypothesis. Complexity 2017, Article ID 4861391, 1-14 (2017)

13. Liu, L., Meng, X.: Optimal harvesting control and dynamics of two-species stochastic model with delays. Adv. Differ. Equ. 2017, 18 (2017)

14. Liu, Q., Jiang, D., Shi, N., Hayat, T., Alsaedi, A.: Stationary distribution and extinction of a stochastic SIRS epidemic model with standard incidence. Physica A 469, 510-517 (2017)

15. Fan, K., Zhang, Y., Gao, S., Wei, X.: A class of stochastic delayed SIR epidemic models with generalized nonlinear incidence rate and temporary immunity. Physica A 481, 198-208 (2017)

16. Rifhat, R., Wang, L., Teng, Z.: Dynamics for a class of stochastic SIS epidemic models with nonlinear incidence and periodic coefficients. Physica A 481, 176-190 (2017)

17. Cai, Y., Kang, Y., Wang, W.: A stochastic SIRS epidemic model with nonlinear incidence rate. Appl. Math. Comput. 305, 221-240 (2017)

18. Zhao, Y., Jiang, D.: The threshold of a stochastic SIS epidemic model with vaccination. Appl. Math. Comput. 243 718-727 (2014) 
19. Capasso, V., Serio, G.: A generalization of the Kermack-McKendrick deterministic epidemic model. Math. Biosci. 42, 43-61 (1978)

20. Xiao, D., Ruan, S.: Global analysis of an epidemic model with nontone incidence rate. Math. Biosci. 208, 419-429 (2007)

21. La, C.A., Valenti, D., Dubkov, A.A., Spagnolo, B.: Dynamics of two competing species in the presence of Lévy noise sources. Phys. Rev. E 82, 011121 (2010)

22. Reynolds, A.M.: Deterministic walks with inverse-square power-law scaling are an emergent property of predators that use chemotaxis to locate randomly distributed prey. Phys. Rev. E 78, 011906 (2008)

23. Stout, J.C., Goulson, D.: The use of conspecific and interspecific scent marks by foraging bumblebees and honeybees. Anim. Behav. 62, 183-189 (2001)

24. Zhao, Y., Yuan, S., Zhang, Q.: The effect of Lévy noise on the survival of a stochastic competitive model in an impulsive polluted environment. Appl. Math. Model. 40, 7583-7600 (2016)

25. Bao, J., Yuan, C.: Stochastic population dynamics driven by Lévy noise. J. Math. Anal. Appl. 91, 363-375 (2012)

26. Leng, X., Feng, T., Meng, X.: Stochastic inequalities and applications to dynamics analysis of a novel SIVS epidemic model with jumps. J. Inequal. Appl. 2017, 138 (2017)

27. Zhang, X., Wang, K.: Stochastic SIR model with jumps. Appl. Math. Lett. 26, 867-874 (2013)

28. Mao, X.: Stochastic Differential Equations and Applications. Horwood Publishing, Chichester (1997)

29. Lipster, R.: A strong law of large numbers for local martingales. Stochastics 3, 217-228 (1980)

30. Kunita, H.: Itô stochastic calculus: its surprising power for applications. Stoch. Process. Appl. 120, 622-652 (2010)

31. Liu, M., Wang, K.: Dynamics of a Leslie-Gower Holling-type II predator-prey system with Lévy jumps. Nonlinear Anal. 85, 204-213(2013)

32. Bao, J., Mao, X.: Competitive Lotka-Volterra population dynamics with jumps. Nonlinear Anal. 74, 6601-6616 (2011)

\section{Submit your manuscript to a SpringerOpen ${ }^{\circ}$ journal and benefit from:}

- Convenient online submission

Rigorous peer review

Open access: articles freely available online

- High visibility within the field

- Retaining the copyright to your article

Submit your next manuscript at $>$ springeropen.com 